Cultures \& Conflits

08 | hiver 1992

Les conflits après la bipolarité

\title{
De la répression à la sécurisation : renouveau policier en Afrique du Sud
}

\section{Philippe Chapleau}

\section{OpenEdition}

1 Journals

\section{Édition électronique}

URL : http://journals.openedition.org/conflits/521

DOI : $10.4000 /$ conflits.521

ISSN : $1777-5345$

Éditeur :

CCLS - Centre d'études sur les conflits lilberté et sécurité, L'Harmattan

Édition imprimée

Date de publication : 6 décembre 1992

ISSN : 1157-996X

Référence électronique

Philippe Chapleau, «De la répression à la sécurisation : renouveau policier en Afrique du Sud »,

Cultures \& Conflits [En ligne], 08 | hiver 1992, mis en ligne le 27 janvier 2003, consulté le 30 mars 2021.

URL : http://journals.openedition.org/conflits/521 ; DOI : https://doi.org/10.4000/conflits.521

Ce document a été généré automatiquement le 30 mars 2021.

Creative Commons License 


\section{De la répression à la sécurisation : renouveau policier en Afrique du Sud}

Philippe Chapleau

Au nom de la Loi et de l'Ordre

1 La grande remise en question des structures de la société sud-africaine n'a pas épargné les forces de l'ordre. Depuis 1990 et la légalisation des partis noirs d'opposition, l'armée (SADF) et la police (SAP) sud-africaines ont dû s'adapter aux nouvelles conditions politiques qui régissent les rapports inter et intra-étatiques dans le sous-continent austral. Alors que la SADF s'est désengagée de ses zones de déploiement angolaises et namibiennes et est entrée dans une semi-léthargie en attendant qu'une décision politique statue sur les missions et les moyens de la future armée sud-africaine, la SAP a vu au contraire une intensification de ses activités.

2 Incontournable, la police sud-africaine l'est certainement. Il serait impensable qu'elle opère un repli similaire à celui de la SADF dans l'actuel climat social et politique. Critiquable et perfectible, elle l'est encore plus. En fait, au cours des trois dernières années, son rôle et l'esprit qui l'anime ont fréquemment été remis en question. La SAP est souvent vue comme un produit de l'apartheid et son instrument majeur de répression. Les opposants ne cessent de rappeler le rôle de certains services de police dans la neutralisation des militants anti-apartheid. Leurs accusations ont été étayées par les confessions d'anciens membres de la police ou d'activistes de réseaux parallèles chargés de la surveillance et de l'élimination de responsables syndicaux, religieux et communautaires au cours de la décennie 80. Les révélations de membres des " Hit Squads " se sont multipliées en 1991 et 1992. La lumière a ainsi pu être faite sur la disparition ou la mort de certains opposants. D'autres confessions ont permis de mieux comprendre les structures de l'appareil policier mis en place sous P.W. Botha et d'esquisser la silhouette des " Contras sud-africains " comme les avait appelés l'universitaire David Webster abattu le 1er mai 1989. Dans un petit ouvrage, Death Squads : Apartheid's Secret Weapons, le journaliste Patrick Laurence donnait dès 1990 
une image plus que convaincante des Escadrons de la mort en RSA et disséquait les activités de deux des plus notoires : le Civil Cooperation Bureau (CCB), dirigé par le chef des forces spéciales de la SADF, le général Webb, et l'unité antiguérilla de Vlakplaas sous les ordres d'un capitaine de la police, Dirk Coetsee. Au cours des deux années suivantes, de nouveaux témoignages ont révélé l'ampleur des opérations des Death Squads et ont contribué à discréditer dramatiquement l'image de la police.

3 La SAP n'a pas seulement été accusée de mener une répression, directe et indirecte, impitoyable. Depuis 1990, on l'accuse également de soutenir les factions noires conservatrices (Vigilantes), en particulier l'Inkatha du chef zoulou Buthelezi, dans leur lutte contre les communautés plus progressistes des townships. Bien qu'aucune preuve formelle n'ait pu être établie pour confirmer une collusion entre la SAP et Inkatha, les témoignages se multiplient dès qu'un massacre a lieu dans un township. L'épisode de la tuerie de Boipatong reste le plus symptomatique. Selon les rescapés du massacre qui a fait une cinquantaine de victimes en juin 1990, les guerriers zoulous qui ont assailli les squatters auraient été acheminés dans des blindés Casspir de la SAP. L'effacement, accidentel selon l'état-major, des bandes des écoutes radio de la nuit du massacre a soulevé des questions encore plus insidieuses sur les responsabilités de la police.

Même si les personnels coupables de ces possibles activités occultes ne constituent qu'une poignée de policiers, c'est l'image globale de la SAP qui en est affectée. Sa crédibilité est donc sérieusement mise en cause au moment même où la situation interne est de plus en plus préoccupante.

La détente en question

5 La légalisation de l'ANC avait marqué une étape importante de la " prétoriastroïka ". En 1990, deux autres pas cruciaux avaient été franchis, l'un en mai avec l'ouverture des entretiens entre l'ANC et le régime blanc, l'autre en août avec l'annonce par Nelson Mandela de la suspension de la lutte armée. Ces progrès devaient mener à la tenue de CODESA (Convention pour une Afrique du Sud démocratique) où le processus de refonte du système allait être négocié. Même si les rapports entre les différents acteurs n'ont pas été exempts de tension, force est de constater que la démocratisation de l'Afrique du Sud est en marche. L'urgence de la résolution de la crise sociale est même occultée par la frénésie des formations politiques à vouloir franchir le seuil critique des premières élections libres.

Ces progrès indéniables ne peuvent cependant pas occulter la dégradation foncière des rapports entre certaines formations politiques noires. La marginalisation, par étapes, $\mathrm{du}$ mouvement zoulou Inkatha par l'ANC, et même à certains égards par le pouvoir blanc, a exacerbé les ambitions et la détermination du chef Buthelezi. Inkatha, avec son million de membres et le soutien de l'administration du Kwazulu, est devenu le fer de lance du chef zoulou qui a réussi à rallier le roi Goodwill Zwelethini autour de l'idée du nationalisme zoulou. Marginalisé à partir du 2 février 1990, Buthelezi a évité de se retrouver dans les limbes de la politique sud-africaine en choisissant de recourir à la violence pour se faire reconnaître et entendre ${ }^{1}$. Exacerbé par de vieilles haines ethniques, le recours à la violence a provoqué un embrasement dans les townships du Natal et du Transvaal, là où la présence zoulou est la plus importante. Même si, aux batailles rangées de 1990 a succédé un climat d'hostilité et de peur, les attentats, les exécutions se multiplient et déstabilisent non seulement la communauté noire mais l'ensemble du processus de rapprochement politico-racial. 
7 Alors que l'extrême droite multiplie les avertissements et les menaces sans passer aux actes, la violence politique la plus meurtrière résulte donc de l'intolérance et du choc de deux idéologies nationalistes noires, l'une progressiste et centralisatrice, l'autre conservatrice et fédéraliste. L'insécurité en chiffres

8 Si les rivalités politiques entre mouvements noirs constituent le facteur majeur de violence et sont de plus en plus médiatisées, la criminalité reste un élément non négligeable de déstabilisation interne.

9 Cette criminalité a des origines aisément identifiables. Depuis 1985, la RSA traverse une crise économique qui se traduit par une inflation annuelle de $15 \%$, par un taux de chômage atteignant 45 \% chez les Noirs et une chute constante du Rand. L'effet de ces difficultés économiques a été amplifié par la sécheresse qui ravage le sous-continent. Tous ces facteurs ont contribué à l'exode rural, au développement de camps de squatters fortement politisés, à la naissance d'un sous-prolétariat et à une exacerbation des frustrations et de l'agressivité au sein des communautés africaines. L'acquisition aisée d'armes, par vol dans les quartiers blancs où la criminalité a dramatiquement augmenté, par prélèvement dans les stocks des mouvements de libération ou par recours au marché noir où l'on trouve aisément des AK 47 mozambicaines, s'est soldée par une recrudescence des attaques à main armée ( + $27 \%$ en 1991) et des embuscades sur les forces de l'ordre. Même les townships ne sont pas épargnés. La pègre noire a profité de l'instabilité créée lors des affrontements entre partisans d'Inkatha et de l'ANC pour se renforcer et s'implanter. Enfin, la présence d'éléments incontrôlés qui lancent des attaques aveugles dans les trains et sur les arrêts de bus contribue à créer un climat de violence sans précédent. En termes de pertes en vies humaines, la période 1990-1992 a été plus meurtrière que les périodes précédentes, pourtant caractérisées par le terrorisme urbain et la répression.

10 Entre juillet 90 et juillet 92, 6907 personnes ont été victimes de la violence politique ; $70 \%$ d'entre elles ont péri dans des affrontements entre factions noires. Si on recensait 39 meurtres pour 100000 habitants en 1990, ce chiffre est passé à 49 en 1991. Quant aux cas de possession illégale d'armes à feu, ils ont augmenté de $92 \%$. En 1991, la SAP a perdu 145 hommes, tués en opérations ou assassinés. Pour 1992, ce chiffre est beaucoup plus lourd ; pour les 10 premiers mois de 1992, 182 policiers ont déjà trouvé la mort.

11 Il est clair que la résolution des conflits traditionnels (lutte armée-répression) n'a pas prévenu une recrudescence de la violence et un accroissement sensible du nombre des victimes. Alors que le pays va entrer dans une période préélectorale où les tensions ne peuvent que s'exacerber, on ne peut donc que craindre une intensification de l'instabilité et des troubles.

12 Cette dégradation de la situation n'a pas été sans inquiéter la classe politique locale. Alors que certains dirigeants voient en la violence un mal nécessaire qui servira à moyen terme leurs desseins, la plupart des responsables ont pris conscience de la gravité de la situation. Mais les initiatives politiques ont pour l'instant échoué. La rencontre Mandela/Buthelezi de janvier 91 ou l'accord de paix d'août 91 n'ont donné aucun résultat tangible. L'insécurité s'accroissant dans les zones blanches et la situation se dégradant dans les townships au point que certains quartiers sont en passe d'échapper au contrôle des forces de l'ordre, l'urgence d'une initiative de sécurité intérieure a obligé les responsables de la police à repenser leur conception du maintien de l'ordre.

L'ISD 
13 Ce qu'on appelle les " forces de sécurité " peuvent être divisées en trois catégories. La première, celle qui dispose des moyens humains les plus vastes (100 000 soldats d'active et plus de 200000 réservistes), regroupe les 4 armes de la SADF : l'armée de terre, la marine, l'armée de l'air et le service de santé. La SADF a récemment vu ses effectifs fondre. La conscription obligatoire pour les Blancs a été réduite de 2 à 1 an et les périodes de réserve des unités de la Citizen Force ou des Kommandos ont été considérablement raccourcies (elles pouvaient atteindre 60 jours par an). Des unités de l'armée de terre sont régulièrement déployées, en soutien de la police, dans les zones de troubles (Unrest Area), mais le manque d'entraînement des soldats aux missions de maintien de l'ordre en zones urbaines et les carences de la SADF en matériels adaptés à ces missions obligent à relativiser les effets de cette assistance.

de route, services de protection des biens administratifs et des personnalités locales. Elles aussi peuvent être amenées à agir de concert avec la police sud-africaine. Leurs tenues, armements et équipements en font en définitive de véritables forces de police auxiliaires.

15 La police sud-africaine (SAP) forme la troisième catégorie. Jusqu'en 1992, ce corps de 114000 hommes (60\% de Noirs) avait la responsabilité des missions de maintien de l'ordre (Riot Units), de police judiciaire (CID), de surveillance des frontières et des voies de communication et de sûreté interne (Security Police). Puisque les personnels civils et les réservistes comptent 25000 personnes, c'est en fait près de 90000 hommes (soit 3 policiers pour 1000 habitants) que peut déployer la SAP. Mais ce chiffre est à préciser, car ces 90000 hommes sont amenés à effectuer tout un éventail de missions, depuis l'îlotage jusqu'à la répression des émeutes. En outre, les services administratifs et logistiques mobilisent un nombre non négligeable de ces personnels (près de $10 \%$ ). Compte tenu des repos, vacances et autres indisponibilités, les moyens en hommes sont en fait très limités. Contrairement à l'impression créée au cours des années 80 , la police sud-africaine manque sérieusement de personnel.

$16 \mathrm{Au}$ cours des 20 dernières années, la doctrine de la SAP s'élaborait autour du concept de polyvalence. Le policier de garde dans un commissariat pouvait aussi bien intervenir pour un vol de voiture que pour un quadrillage dans un township. Tous les deux ans, il prenait part à un tour opérationnel aux frontières dans le cadre de la lutte antiguérilla (COIN). Quant aux fameuses Riot Units, elles n'étaient constituées que de façon ad hoc, selon les disponibilités en hommes. Ce concept était, selon le général Durring qui commandait les forces anti-émeutes en 1989, " inaltérable ". La mise sur pied d'unités spécialisées n'était pas envisagée, ni envisageable. Cette tactique de fortune avait ses revers. La polyvalence signifiait souvent, la multiplication des tâches, d'où une réelle fatigue physique et nerveuse chez des hommes travaillant par " shift " de 12 heures au minimum, un manque d'entraînement pour des missions spécifiques et l'incapacité d'identifier les personnels les plus aptes à certains types de missions. En termes d'équipement, la polyvalence restait aussi le maître mot. Le blindé Casspir conçu pour la lutte antiguérilla devenait le cheval de bataille de la SAP ; quant aux armes, elles se sont toujours limitées aux fouets, aux pistolets Berreta, aux R1 (FN belges) et aux fusils à pompe. Le maintien de l'ordre était donc effectué avec de l'armement offensif. Toutes ces carences expliquent le nombre élevé de victimes lors des interventions policières des décennies 70 et 80 . 
17 L'évolution politique, la persistance des accusations lancées contre la SAP et la détérioration de la situation dans les townships ont poussé les responsables de la police sud-africaine à réviser leur stratégie. En 1991, un effort avait été fait au niveau des personnels. 2500 auxiliaires (kitskonstabels) avaient été recrutés, 600 réservistes rappelés et 800 civils affectés à des tâches administratives pour libérer un nombre égal de policiers. Le budget de la police avait alors été porté à 5,6 milliards de Rand, soit 21 $\%$ de plus qu'en 1990. La situation restait cependant critique dans les townships où la population n'avait plus qu'une confiance relative en la police et où les affrontements intra-communautaires ne perdaient pas de leur intensité. Pour y remédier, il a été décidé de créer une unité spécialisée dans le maintien de l'ordre et de lui donner une certaine indépendance à l'égard des structures traditionnelles de la SAP. Commandée par un général de division (le général Swart, ancien responsable de la police de Soweto de 89 à 91), l'Internal Stability Division dépend directement du Commissioner of Police et du ministre de la Loi et de l'Ordre. L'ISD a été officiellement créée le 1er janvier 1992. Sa mission est double : prévention et stabilisation. Les unités de l'ISD (ISU), 23 pour l'instant, sont déployées dans les zones à risques (High Intensity Areas) où $86 \%$ des violences ont lieu : le PWV (Johannesburg, Prétoria, Vereeniging), le Natal, la péninsule du Cap et la côte de la province du Cap. Si à terme, l'ISD peut compter sur 17500 hommes, elle ne dispose pour l'instant que de 6000 hommes.

Pour projeter une image régénérée et démarquer l'ISD du reste de la SAP, qui progressivement doit réintégrer le rôle traditionnel des forces de police et abandonner totalement à l'ISD le contrôle des foules et les missions de stabilisation dans les townships, tous les véhicules des ISU ont été repeints. Le jaune SAP a été remplacé par un vert chlorophylle. La tenue SAP, chemise bleu ciel et pantalon de treillis bleu marine, a été abandonnée, mais les contraintes budgétaires n'ont pas permis de doter les personnels de l'ISD d'une tenue originale, identifiable et adaptée ! L'ISD a donc dû, temporairement selon les officiers de l'état-major de l'ISD, recourir aux tenues camouflées utilisées par la SAP lors de ses déploiements en zones opérationnelles (Namibie, frontières du Botswana...). Un béret camouflé remplace par contre la casquette. Bien que les motivations budgétaires soient légitimes, le choix de cette tenue reste critiquable; elle projette une image trop militaire, trop agressive. Elle n'en reste pas moins bien adaptée aux conditions opérationnelles: résistante, bien conçue, discrète de nuit...

19 L'armement reste standard : Beretta et fusils à pompe. Mais le R1 a été remplacé par la version commando du fusil d'assaut $\mathrm{R} 4$ de la SADF ; le R5 a une crosse repliable et un canon court qui en font l'arme idéale en cas d'embuscade pour contrer les tirs d'AK 47 des assaillants. Des équivalents du M 79 américain, les " stoppers " de $37 \mathrm{~mm}$, sont utilisés pour le tir des projectiles en caoutchouc, pour la neutralisation individuelle ou la dispersion des manifestants. Mais la dotation en armes des patrouilles (un blindé et 5 hommes) reste en fait à la discrétion du chef de patrouille. Ainsi, 5 hommes en mission à Sebokeng, en octobre, disposaient de 2 R5, 2 fusils à pompe, 2 " stoppers ", d'un pistolet-mitrailleur Uzi et d'une mitrailleuse légère! L'éventail des menaces explique l'éventail des armes en dotation.

20 Les véhicules de l'ISD sont pour la plus grande partie des blindés, les camionnettes et les 4 x 4 n'étant plus utilisés dans les zones à risques. Ces blindés sont de deux types : Casspir et Nyala. Le Casspir est devenu non seulement une silhouette familière dans les townships mais aussi l'un des symboles de la répression. Ce blindé capable de résister 
aux mines, rustique et efficace est progressivement remplacé par un nouveau type de véhicules d'intervention: un blindé léger à roues, le Nyala. Tout-terrain, capable de transporter 8 hommes équipés, il est destiné au contrôle des foules et à l'assistance rapide en milieu urbain. Son blindage lui permet de résister aux tirs de 12,7. Il est équipé d'un système de climatisation, d'une réserve d'eau potable, d'un lot de bord outillage, d'un lot de bord médical et de deux portes latérales qui permettent une sortie plus aisée et plus rapide qu'à bord des Casspir.

21 La sélection et la formation des personnels de l'ISD ont été particulièrement étudiées. Si l'on excepte l'encadrement, la moyenne d'âge est de 21 ans. Il est apparu nécessaire d'écarter les policiers trop marqués par leur passé, leur formation et leurs expériences. Psychologiquement, ces hommes auraient eu du mal à s'adapter aux mission de l'ISD où le contact et la communication avec les habitants des townships sont primordiaux. Ce sont donc de jeunes policiers frais émoulus des " collèges de police " qui intègrent l'ISD après avoir subi des tests psychologiques. En fait, tous les sous-officiers et les officiers qui ont rejoint l'ISD ont également été évalués. Plusieurs centaines de postulants ont été écartés et au moins 200 policiers ont été retirés du service pour cause de " stress ". Ce processus d'évaluation a été mis sur pied par une cellule de spécialistes, psychologues, sociologues et chercheurs en défense, qui ont analysé l'image projetée par la SAP, les attentes des Noirs et les objectifs de la nouvelle unité.

Tous les personnels suivent une formation spécialisée dans deux nouveaux camps au nord de Prétoria, à Jakalsdans et à Verdrag. L'école de lutte antiguérilla de Maléoskop est également utilisée par l'ISD.

Un cours de remise à niveau est obligatoire. Parmi les rubriques abordées: droit, survie, relations humaines, balistique, maintien de l'ordre, communication... Un " Advanced Course " d'une semaine permet d'approfondir ces thèmes et d'aborder l'ethnologie, les techniques de négociations, la psychologie des foules, les relations avec la presse... Deux autres formations sont dispensées : un stage pour les chefs de groupe et une formation antiterroriste destinée aux hommes des " Reaction Squads " des ISU. Toute cette formation s'articule autour du concept de " Participative Management " pour développer l'autonomie et le sens des responsabilités des policiers. L'entrainement se poursuit au sein des ISU où des instructeurs-tournant poursuivent la formation et testent de nouvelles tactiques. La participation d'intervenants extérieurs est à noter : des universitaires, des responsables locaux et des journalistes donnent des conférences. L'instruction perd ainsi de sa rigidité et est mieux adaptée au profil souhaité pour les personnels qui font de la prévention et non plus de la répression et pour qui le maintien de l'ordre passe par la médiation.

En septembre 1992, 23 unités avaient déjà été constituées et déployées. Les moyens en hommes des ISU varient : de 150 à 350 hommes, avec une exception, l'unité 19, basée à Prétoria, forte d'environ 900 hommes et qui constitue la FAR de l'ISD et bénéficie d'un soutien logistique permanent de l'armée de l'air.

Premier bilan

Il était urgent que soit créée en Afrique du Sud une unité spécialisée dans le maintien de l'ordre et bénéficiant d'une volonté politique de promotion de l'apaisement. Les errements des responsables pendant 20 ans ont jeté un très lourd discrédit sur la SAP qui a dû employer une partie de ses moyens pour des tâches éminemment politiques et répressives et qui a perdu la confiance des Noirs qui la percevaient comme un instrument totalitaire, ainsi que celle des Blancs qui lui reprochaient de ne pas enrayer 
la hausse de la criminalité. La formation de l'ISD est symptomatique des transformations actuelles en RSA et constitue un nouveau gage de la bonne volonté des dirigeants sud-africains de pacifier les zones troubles sans recourir à la répression.

Le progrès est indiscutable mais des difficultés persistent. En termes de moyens humains, l'ISD est en sous-dotation. Les carences en personnels obligent à des " shift " de 12 heures pendant quatre jours consécutifs. L'unité 1 ne disposait en octobre que de 45 hommes par " shift " soit 9 patrouilles pour les townships de Mamelodi, Attridgeville et Shoshanguwe. L'unité 5 à Sebokeng, une des zones les plus dures, ne disposait que de 70 hommes par tour d'opérations; son effectif a été porté en octobre à 315 hommes, ce qui allait permettre de déployer 120 hommes par " shift ".

L'utilisation des tenues camouflées, explicable pour cause de contraintes budgétaires, projette une image beaucoup trop agressive de l'ISD et annule l'impression voulue de détente et de pacification. Le port trop ostensible des armes constitue également un facteur négatif. Il est vrai que les nombreuses embuscades et la détermination des assaillants obligent les ISU à disposer d'un armement adéquat pour la riposte. Mais une rapide évaluation de la situation devrait permettre aux patrouilles de juger de la gravité de la situation et de ne s'équiper qu'avec l'armement de circonstances.

Dans certaines zones, l'ISD n'a pas su se démarquer suffisamment de la SAP et est encore vue comme une force d'occupation par les habitants marqués par 20 ans d'états d'urgence et de brutalités policières. Les masses noires sont traumatisées et la violence intra-communautaire n'atténue en rien ses frustrations et son ressentiment. En fait, l'ISD évolue sur un terrain qui n'a pas évolué en dépit de la fin de l'apartheid. Socialement et humainement, la libéralisation n'a encore rien apporté aux habitants des townships. Pire, les rivalités entre factions noires ont convaincu que la violence constitue le paramètre final de la résolution de toute crise et que les forces de l'ordre sont par essence contre les résidents. S'il existe une cure, elle n'est que socioéconomique. Les townships ne peuvent pas se " ghettoïser " davantage. Leurs habitants ne peuvent plus être considérés comme des laissés-pour-compte. Si cette situation n'était pas redressée à court terme, on assisterait à un phénomène grave : le sousprolétariat noir - squatters, chômeurs et migrants ruraux - prendrait le contrôle des townships alors que la frange plus aisée des Noirs salariés quitterait ses lieux de résidence traditionnels pour gagner les zones grises en périphérie des quartiers blancs. L'instabilité deviendrait incontrôlable dans les ghettos où les forces de l'ordre n'auraient que le choix entre le retrait ou la répression.

Il est donc vital que l'urgence du redressement social ne soit plus ignorée par les politiciens qui ont souvent eu tendance à s'en remettre à la police pour faire régner l'ordre au lieu d'aborder de front les difficultés. C'est donc sur une stratégie d'apaisement que doivent se pencher les technocrates locaux tout en sachant qu'aucune mesure ne peut globalement résoudre la crise. La formation de l'ISD constitue une décision tardive mais louable; il faut massivement déployer une force de police spécialisée dans les ghettos noirs, une force de police animée du désir de faire évoluer les mentalités et d'assurer la protection des citoyens. Les appels de certains groupes d'opposition pour un retrait des forces de sécurité et leur remplacement par des unités d'autodéfense (SDU de l'ANC par exemple) relève de la provocation. Les factions noires et blanches n'attendent qu'un geste de faiblesse du pouvoir pour jouer leur propre jeu et recourir à l'affrontement pour faire triompher ou survivre leurs vues. 


\section{NOTES}

1. Philippe Chapleau, " Les émeutes en Afrique du Sud : la stratégie de Buthelezi ", Cultures \& Conflits,, Emeutes urbaines : le retour du politique, n5, L'Harmattan, printemps 1992.

\section{RÉSUMÉS}

Les bouleversements que connaît actuellement l'Afrique du sud, n'ont pas épargné la SAP (Police Sud Africaine), considérée par la population noire comme un instrument et par la population blanche comme incapable de stopper l'augmentation de la criminalité. Pour faire face à cette perte de crédibilité, alors que la situation interne empire, le gouvernement a créé le 1er janvier 1992, une unité spécialisée dans le maintien ordre, l'ISD (Internal Stability Division). La mission de l'ISD est double : prévention et stabilisation. Elle doit donc se démarquer de la SAP en limitant cette dernière dans un rôle plus traditionnel, et satisfaire à la volonté du gouvernement de pacifier les ghettos sans pour autant recourir à la répression. Pour Philippe Chapleau l'ISD demeure une création tardive même si la décision qui l'a instituée est louable.

Current upheavals in South Africa have not spared the South African Police (SAP), viewed by the Black population as an instrument of repression, and by Whites as inefficient in controlling the sweeping tide of crime. In order to combat this loss of credibility in a worsening context, the Government created a new specialised force on the 1st of January 1992, the ISD (Internal Stability Division). The mission of this new force is dual : prevention and stabilisation. It distinguishes itself from the SAP, the latter playing a more traditional role in assuming the task of pacifying without recourse to repression. The author commends this governmental decision, but deplores the fact that it came so late.

\section{INDEX}

Index géographique : Afrique australe, Afrique du Sud (R.S.A.)

Mots-clés : émeutes, police, répression

Thèmes : ANC 